\title{
Case Report: Asymptomatic Osteosarcoma
}

\author{
Reiko Yamaguchi' ${ }^{1}$, Seiichi Matsumoto ${ }^{1}$, Keisuke Ae1, Taisuke Tanizawa', Tabu Gokita1, \\ Keiko Hayakawa1, Yuki Funauchi' ${ }^{1}$, Munehisa Kito', Hirotada Ishii' ${ }^{2}$, Noriko Motoi ${ }^{3}$ \\ ${ }^{1}$ Department of Orthopaedic Surgery, Cancer Institute Hospital, Tokyo, Japan \\ ${ }^{2}$ Ishii Orthopaedic Clinic, Tokyo, Japan \\ ${ }^{3}$ Division of Pathology, Cancer Institute Hospital, Tokyo, Japan \\ Email: rei-921-Iuna@hotmail.co.jp
}

Received 18 November 2015; accepted 22 December 2015; published 25 December 2015

Copyright (C) 2015 by authors and Scientific Research Publishing Inc.

This work is licensed under the Creative Commons Attribution International License (CC BY).

http://creativecommons.org/licenses/by/4.0/

(c) (i) Open Access

\begin{abstract}
Osteosarcomas are primary malignant tumors that are characterized by the production of osteoid or immature bone. However they are the most common primary malignant tumor of bone in adolescents and young adults, the diagnosis of osteosarcoma is often delayed. It is because of the rarity of population, the variety of findings, nonspecific symptoms and so on. A 46-year-old male patient had left knee pain for 3 months without any symptoms in his right knee. At a nearby clinic, $\mathrm{X}$-ray of his healthy side was taken by chance. The irregular bone formation was however seen in the metaphysis of the distal femur with spreading into the soft tissues. No abnormal findings were pointed out at his left side knee. A diagnosis of pleomorphic sarcoma was made by incisional biopsy. Pulmonary CT showed multiple nodules which were considered as multiple lung metastases. He was diagnosed as right femoral sarcoma, clinical stage IV (T1N0M1a) by TNM classification. He underwent preoperative chemotherapy, followed by limb-saving operation. No preoperative complaints were found in his left knee. Final diagnosis of surgically resected specimen was osteoblastic osteosarcoma which showed neoplastic osteoid formation within pleomorphic malignant cells. However, osteosarcomas occurred in adult populations are sometimes occurred as a secondary neoplasms, he had a history of diabetes but no other medical history, such as radiation therapy for a solid cancer, Paget's disease, other benign bone lesions or inherited conditions. As far as our knowledge, we believe that this is a first case report of asymptomatic incidental osteosarcoma.
\end{abstract}

\section{Keywords}

Incidental Osteosarcoma, Asymptomatic Osteosarcoma, De Novo Osteosarcoma

\section{Introduction}

Osteosarcomas are uncommon malignant bone tumors, which are characterized by the production of osteoid or

How to cite this paper: Yamaguchi, R., Matsumoto, S., Ae, K., Tanizawa, T., Gokita, T., Hayakawa, K., Funauchi, Y., Kito, M., Ishii, H. and Motoi, N. (2015) Case Report: Asymptomatic Osteosarcoma. Case Reports in Clinical Medicine, 4, 369-375. 
immature bone by the malignant cells. The incidence has a bimodal distribution with peaks in early adolescence and in elderly.

In children, osteosarcomas account for approximately 3 percent of childhood cancers overall. The peak incidence is during the adolescent growth spurt.

In adults, osteosarcomas are often considered as secondary neoplasms. About one-third of osteosarcomas in adults arise in a background of Paget's diseases of bone or previous cancer. Several risk factors of osteosarcoma in adults are recognized, such as Paget's disease, inherited conditions, benign bone tumor, and prior irradiation or chemotherapy.

Almost all osteosarcoma patients complain of symptoms related to their lesion at the first medical visit. Physical findings are regional pain, palpable mass, restricted movement of joint and others. A previous study about complaints of malignant bone tumors reported that $85 \%$ patients had pain related strain, $21 \%$ had pain at night, $47 \%$ had the onset of symptoms related to minor trauma and 39\% had a palpable mass.

We experienced a rare case of asymptomatic osteosarcoma who was diagnosed accidentally. Here, we report a rare case of incidental osteosarcoma.

\section{Case Report}

A 46-year-old man was referred to our hospital because of irregular bone formation in his right femur.

He did not suffer any pain until 7 months before hospital admission, due to his left knee pain. He had not visited a medical institution for 6 months because he had no difficulties in daily life. He saw his primary care provider half a year after the pain had begun. He reported left knee pain, but no symptoms in his right leg. A radiograph of his healthy side, the right knee, was taken by mistake. The result showed irregular bone formation in the right femur. However, no symptoms such as palpable mass, atrophy of muscle, fever and weight loss were found.

His medical history included only diabetes mellitus, without history of benign bone lesion, Paget's disease, inherited conditions, and prior irradiation or chemotherapy. He had no family history of malignancy.

His vital signs were within normal limits with a blood pressure of $122 / 87 \mathrm{mmHg}$, a pulse of 82 beats per minute, a temperature $36.8^{\circ} \mathrm{C}$, and a room air oxygen saturation of $99 \%$. The weight was $104.6 \mathrm{~kg}$, height 165.2 $\mathrm{cm}$, and body mass index 38.3. Physical examination showed normal conditions other than left knee pain. The serum alkaline phosphatase level was not high (294U/l), and the levels of total protein, albumin, lactate dehydrogenase, blood urea nitrogen, sodium, potassium, chloride and C-reactive protein were within normal limits. Liver and renal function were normal. According to the screening tests, the serum levels of tumor markers, including carcinoembryonic antigen, squamous cell carcinoma antigen, pro gastrin-releasing peptide and cytokeratin 19 fragment were not high. The complete blood count was normal. The activated partial-thromboplastin time, the prothrombin time and the international normalized ratio were within normal range.

His leg radiograph revealed irregular bone formation in the metaphysis of the distal femur, which spread to soft tissues (Figure 1). There were no abnormal findings in the left side. Magnetic resonance imaging (MRI) showed that a mass occupied the same part of the right femur $(2.7 \mathrm{~cm}$ and $3.1 \mathrm{~cm}$ and $3.0 \mathrm{~cm}$ in diameter) where the irregular bone formation was seen on the radiograph, and that soft tissues around the lesion were edematous (Figure 2). Bone scintigraphy showed an abnormal uptake on the distal part of the right femur (Figure 3). Computed tomography (CT) revealed an indistinct osteolytic lesion in the medial distal femoral metaphysis with erosion of cortex and solid periosteal reaction $(1.7 \mathrm{~cm}$ and $4.0 \mathrm{~cm}$ and $2.8 \mathrm{~cm}$ in diameter) (Figure 4). Pulmonary CT showed multiple masses of various sizes but no lymphadenopathy was found (Figure 5). Incisional biopsy was done, and the lesion was diagnosed as pleomorphic sarcoma.

He had received neoadjuvant chemotherapy, combination with cisplatin and adriamycin. After one course of the regimen, he could not continue it due to renal dysfunction. We performed extraarticular resection of the left femoral tumor and arthroplasty.

After the operation, CT showed that pulmonary metastases had increased. He received chemotherapy again. A regimen of Carboplatin caused renal dysfunction. He continued the chemotherapy, Doxorubicin and Cyclophosphamide by turns. After six courses, pulmonary CT revealed the spread of metastases. He complained of headache and diplopia, and MRI showed the multiple metastases of brain. He received radiation therapy for brain. Two years later after the operation, he died of respiratory failure caused by tumors. 


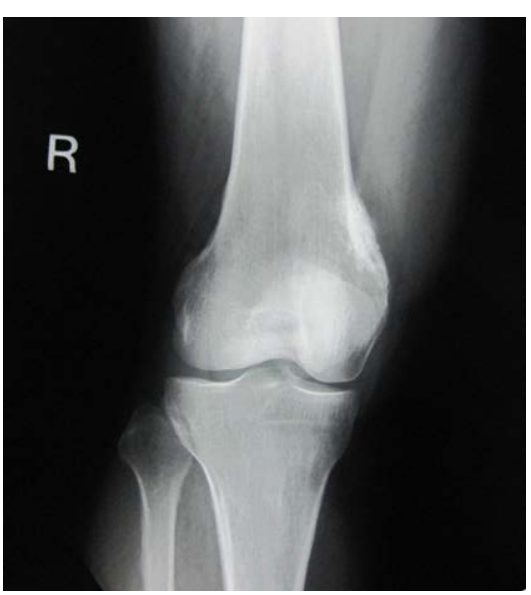

Figure 1. AP plain radiograph of the right knee. There is a lytic lesion in the distal femur with a sclerosed cortex and periosteal reaction. (Arrow)

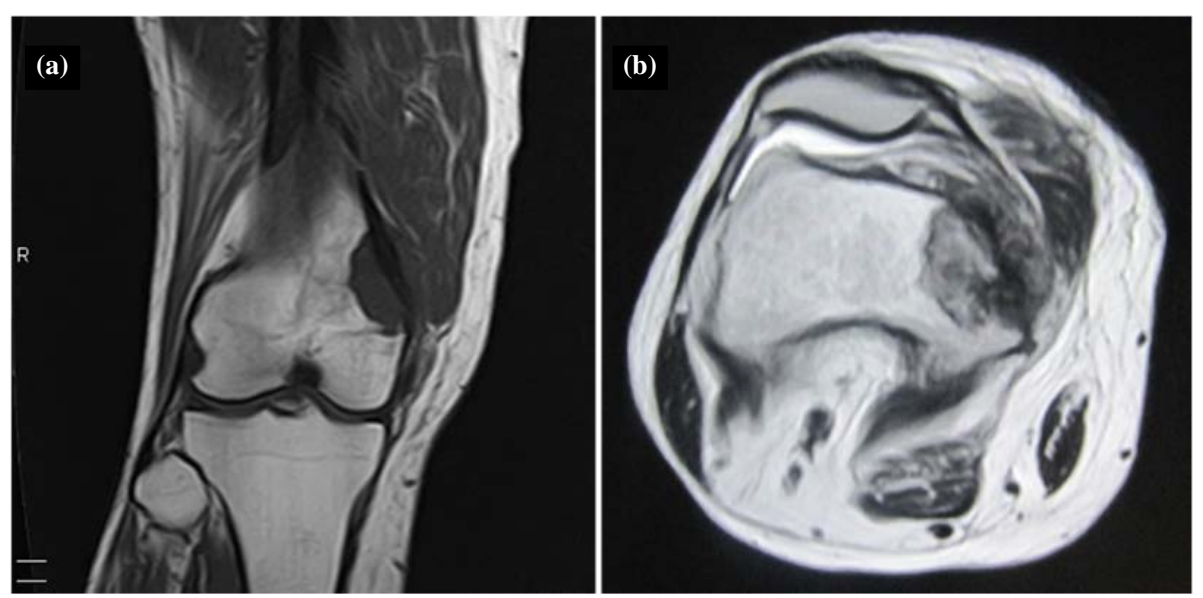

Figure 2. MR images of the right knee. (a) Coronal T1-weighted MR image shows focal marrow of decreased signal intensity in the distal femur and periosteum. (Arrow); (b) Axial T2-weighted MR image shows a bone marrow lesion of mixed signal intensity, eroding soft tissue anterior to the femur. (Arrow).

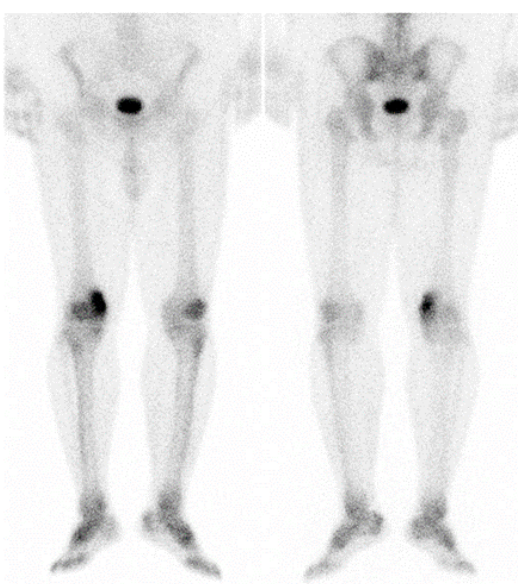

Figure 3. Image of bone scintigraphy of the lower extremities. Bone scintigraphy shows an eccentric radionuclide uptake on the distal part of the right femur. There is no other abnormal uptake. (Arrow) 


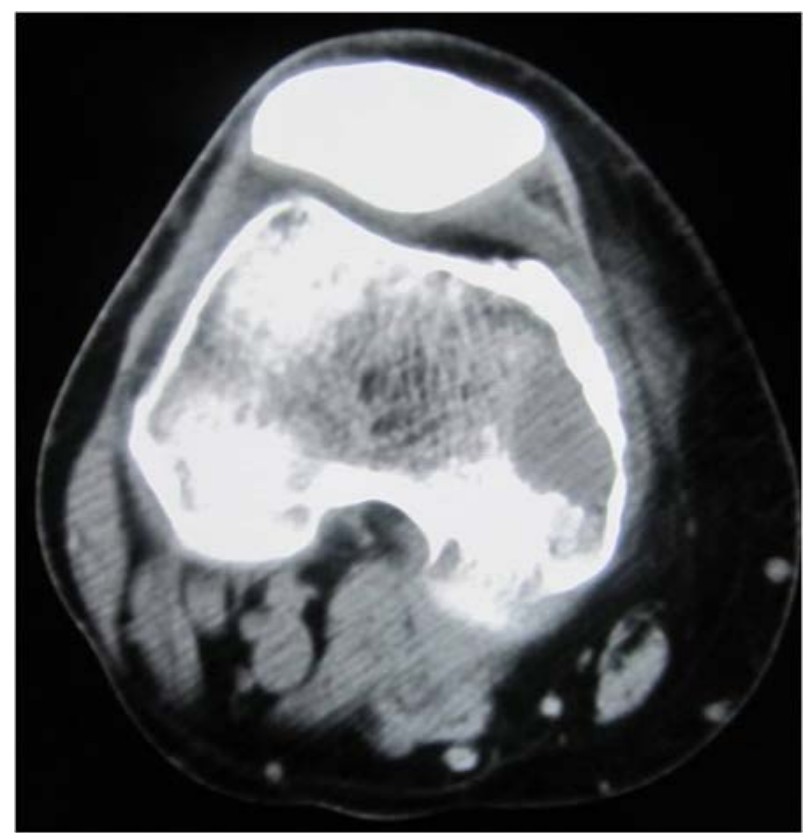

Figure 4. CT image of the right knee. CT shows an indistinct lytic lesion in the medial distal femoral metaphysis.

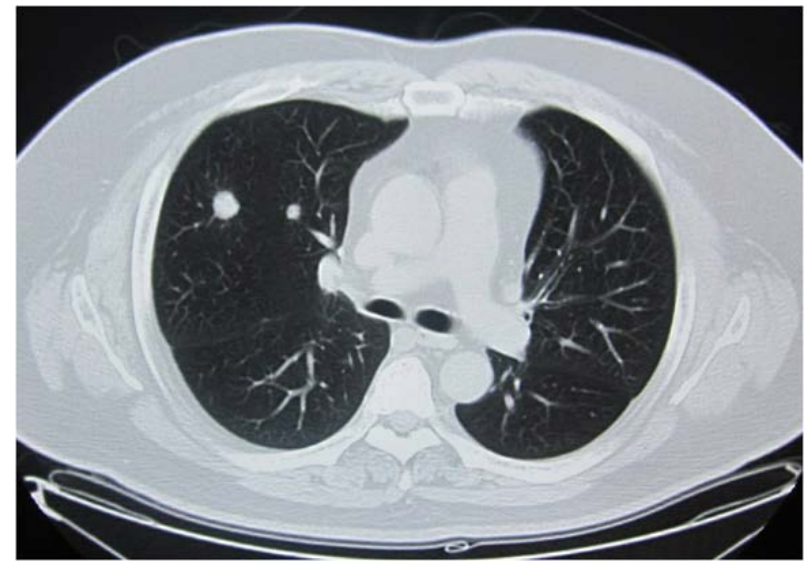

Figure 5. Pulmonary CT. Pulmonary CT shows multiple masses, which are suspected as pulmonary metastasis. (Arrow)

\section{Pathological Findings}

\subsection{Incisional Biopsy}

Histological findings of biopsy specimen showed proliferation of pleomorphic atypical spindle cells with neoplastic osteoid formation. Immunohistochemical staining of the tumor cells showed positive for desmin, alpha-smooth muscle actin (SMA), muscle-specific actin (HHF35), and focally for S100, but negative for cytokeratin(CK) AE1/3, myogenin and myogenic differentiation 1 (MyoD1). Ki67/MIB1 labeling index was over 30 percent, score 3 (Figure 6). The diagnosis of osteoblastic osteosarcoma, grade 3 was made.

\subsection{Resected Specimen}

A surgically resected specimen showed $5.0 \times 4.9 \times 3.6 \mathrm{~cm}$-sized tumor, located in the metaphysis of the right femur with extension around the soft tissue. The histological findings of this lesion were similar to the previous biopsy specimen, e.g. osteogenic sarcoma. The tumor composed of pleomorphic undifferentiated sarcomatous 
area, conventional osteoblastic osteosarcoma (grade 3 - 4) and low grade fibroblastic osteosarcoma (grade 1 - 2) (Figure 7). Minute foci of chondroblastic area were also found. The sarcoma cells directly invaded to the extraskeletal connective tissue, breaking through the periosteum. There was small area of necrosis and fibrous area within the tumor.

\section{Discussion}

Patients of osteosarcoma generally have some symptoms such as pain or palpable mass [1]-[3]. This case was found although the patient did not suffer any pain on his pathological side. It is a rare case of osteosarcoma presenting as 'incidentaloma'.

Widhe (2000) reported initial symptoms and clinical features in osteosarcomas [1]. According to the study, physical findings, at the first medical visit, of osteosarcomas are local tenderness (93\%), palpable masses (39\%), painful movement of joint (39\%), limp (31\%) and restricted movement of joint (23\%), atrophy of muscle (5\%) and fever (3\%). Systemic symptoms such as weight loss, malaise and dullness are uncommon. O. Sneppen (1984) reported presenting symptoms of osteosarcoma [4]. They reported that 62 (74\%) of 84 patients had regional pain, 21 patients (25\%) had observed swelling, and in 4 patients (5\%) a pathologic fracture was the initial sign. Thus patients generally have some disorders at their first medical visit. We have no idea why his left knee had pain instead, but perhaps he had protected his right knee unconsciously.

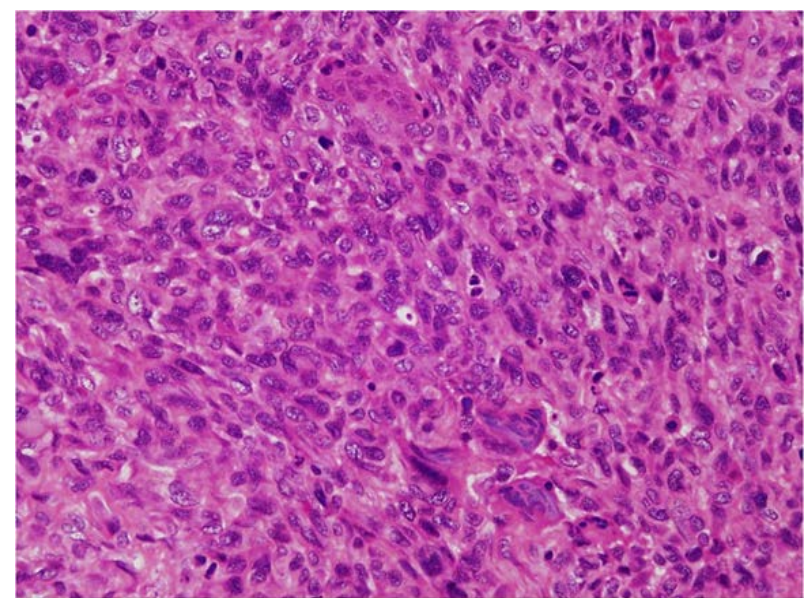

Figure 6. Slide of the incisional biopsy (hematoxylin and eosin). Slide shows hyperplasia of pleomorphic atypical spindle cell sarcoma with neoplastic osteoid.

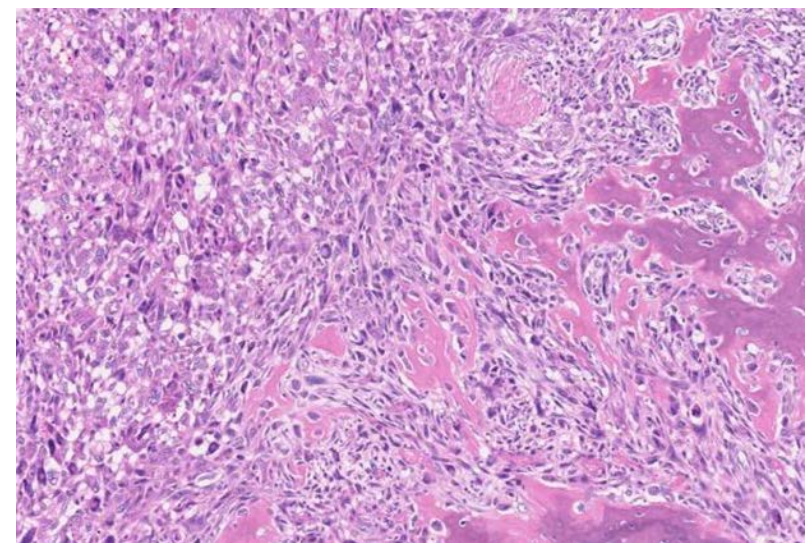

Figure 7. Slide of surgically resected specimen. Slide shows an area composed of conventional osteoblastic osteosarcoma (grade 3 - 4). 
As far as we know, there has been no report of asymptomatic osteosarcoma, but a case of Ewing's sarcoma without pain has been reported. Edwin Choy (2009) reported a case of 23-year-old male with cough and, hoarseness that had developed a month beforehospital admission [5]. He was transferred to a hospital for the symptoms and abnormalities on chest imaging. After careful examination, he was diagnosed with Ewing's sarcoma in the right iliac bone, while he did not suffer any pain or other symptoms as primary lesion. We could not find a case report of pulmonary metastasis leading to the first symptoms. However, Haga T. (2011) reported an autopsy case of extraskeletal osteosarcoma arising from the mediastinum, where the first symptoms were dyspnea and palpitation [6].

Mayordomo JI (1993) showed neoplasm of unknown primary site(NUPS) [7]. They reviewed 1656 autopsies performed on adults in their institution from 1974 to 1990, and 43 cases of NUPS were found. As a result, clinical presentation included general deterioration (73\%), digestive symptoms (58\%), liver enlargement (58\%), abdominal pain (56\%), respiratory symptoms (45\%), ascites (26\%), and node enlargement (16\%). Pathologic diagnoses at autopsy were: 23 adenocarcinomas (53\%), 3 squamous carcinomas (5\%), 5 undifferentiated carcinomas, and 12 miscellaneous tumors. There was no case of sarcoma reported.

K. Godley (2008) showed the features of patients with a malignant primary bone tumor of the femur with a pathological fracture [8]. According to the study, $26 \%$ of them were in the distal femur and the most common histological diagnoses were osteosarcoma. The median survival was 12 months.

F. Gouin (2008) reported the diagnostic difficulties about osteosarcoma over 40 years of age [9]. This showed that some patients had been referred after inadequate treatment or unsuitable surgeries.

Thus, the diagnosis of osteosarcoma in adults is difficult. In our case, if he had not diagnosed by chance, he might be misdiagnosed leading to non-optimal treatment or be diagnosed by a pathological fracture, respiratory or other symptoms after he got worse or when he suffered pathological fracture.

Mark D. Murphey (1997) pointed that conventional osteosarcoma were aggressive lesions with rapid doubling times and therefore were often large at the time of diagnosis [10]. We think that he was asymptomatic because he had no external injury triggering fracture, the lesion was relatively small and his obesity hided the mass.

\section{Conclusions}

We experienced a rare case of asymptomatic osteosarcoma. We found no report of osteosarcoma presenting as "incidentaloma". In almost all cases, osteosarcoma was not diagnosed until symptoms appeared.

The diagnosis of osteosarcoma in adult populations is difficult, and some have been misdiagnosed or diagnosed after progress unlike this case.

\section{References}

[1] Widhe, B. and Widhe, T. (2000) Initial Symptoms and Clinical Features in Osteosarcoma and Ewing Sarcoma. The Journal of Bone \& Joint Surgery, 82, 667-674. http://jbjs.org/content/82/5/667.long

[2] Wang, L.L., Chintagumpala, M., Gebhardt, M.C. (2014) Osteosarcoma: Epidemiology, Pathogenesis, Clinical Presentation, Diagnosis, and Histology.

http://www.uptodate.com/contents/osteosarcoma-epidemiology-pathogenesis-clinical-presentation-diagnosis-and-histol ogy

[3] Hornicek, F.J. (2015) Bone Sarcomas: Preoperative Evaluation, Histologic Classification, and Principles of Surgical Management.

http://www.uptodate.com/contents/bone-sarcomas-preoperative-evaluation-histologic-classification-and-principles-of-s urgical-management

[4] Sneppen, O. and Hansen, L.M. (1984) Presenting Symptoms and Treatment Delay in Osteosarcoma and Ewing’s Sarcoma. Acta Radiologica Oncology, 23, 2-3. http://dx.doi.org/10.3109/02841868409136005

[5] Choy, E., Digumarthy, S.R. and Koplin, S.A. (2009) Case 36-2009: A 23-Year-Old Man with Cough, Hoarseness, and Abnormalities on Chest Imaging. The New England Journal of Medicine, 361, 2080-2087. http://www.nejm.org/doi/full/10.1056/NEJMcpc0907804

[6] Haga, T., Fukuoka, M., Morita, M., Cho, K. and Ozawa, S. (2011) An Autopsy Case of Extraskeletal Osteosarcoma Arising in the Mediastinum. Nihon Kokyuki Gakkai Zasshi, 49, 237-240.

[7] Mayordomo, J.I., Guerra, J.M., Guijarro, C., García-Prats, M.D., Gómez, A., López-Brea, M., et al. (1993) Neoplasms of Unknown Primary Site: A Clinicopathological Study of Autopsied Patients. Tumori, 79, 321-324.

http://europepmc.org/abstract/MED/8116074 
[8] Godley, K., Watts, A.C. and Robb, J.E. (2008) Pathological Fracture of the Femur as a Presenting Feature of Primary Bone Tumor. The Bone \& Joint Journal, 90, 400. http://scm.sagepub.com/content/56/1/5.long

[9] Gouin, F., Touré, M., Roland, F., Moreau, A. and Bertrand-Vasseur, A. (2004) Osteosarcoma in Subjects over 40 Years of Age: A Difficult Diagnostic Entity with a Serious Prognosis. The Bone \& Joint Journal, 86-B, 65 http://www.bjjprocs.boneandjoint.org.uk/content/86-B/SUPP_I/65.3

[10] Murphey, M.D., Robbin, M.R., McRae, G.A., Flemming, D.J., Temple, H.T. and Kransdorf, M.J. (1997) The Many Faces of Osteosarcoma. RadioGraphics, 17, 1205-1231. http://pubs.rsna.org/doi/pdf/10.1148/radiographics.17.5.9308111 